\title{
A review on processing techniques of bast fibers nanocellulose and its polylactic acid (PLA) nanocomposites
}

\begin{abstract}
The utilization of nanocellulose has increasingly gained attentions from various research fields, especially the field of polymer nanocomposites owing to the growing environmental hazardous of petroleum based fiber products. Meanwhile, the searching of alternative cellulose sources from different plants has become the interests for producing nanocellulose with varying characterizations that expectedly suit in specific field of applications. In this content the long and strong bast fibers from plant species was gradually getting its remarkable position in the field of nanocellulose extraction and nanocomposites fabrications. This review article intended to present an overview of the chemical structure of cellulose, different types of nanocellulose, bast fibers compositions, structure, polylactic acid (PLA) and the most probable processing techniques on the developments of nanocellulose from different bast fibers especially jute, kenaf, hemp, flax, ramie and roselle and its nanocomposites. This article however more focused on the fabrication of PLA based nanocomposites due to its high firmness, biodegradability and sustainability properties in developed products towards the environment. Along with this it also explored a couple of issues to improve the processing techniques of bast fibers nanocellulose and its reinforcement in the PLA biopolymer as final products.
\end{abstract}

Keyword: Bast fibers; Biopolymer; Nanocellulose; Nanocomposites; Polylactic acid; Processing technique 